\title{
Sunspots at centimeter wavelengths
}

\author{
Mukul R. Kundu' ${ }^{1}$ and Jeongwoo Lee ${ }^{2}$ \\ ${ }^{1}$ Department of Astronomy, University of Maryland, \\ College Park, MD 20742, U.S.A. \\ ${ }^{2}$ Physics Department, New Jersey Institute of Technology, \\ Newark, NJ 07102, U.S.A. \\ email: leej@njit.edu
}

\begin{abstract}
The early solar observations of Covington (1947) established a good relation between $10.7 \mathrm{~cm}$ solar flux and the presence of sunspots on solar disk. The first spatially resolved observation with a two-element interferometer at arc min resolution by Kundu (1959) found that the radio source at $3 \mathrm{~cm}$ has a core-halo structure; the core is highly polarized and corresponds to the umbra of a sunspot with magnetic fields of several hundred gauss, and the halo corresponds to the diffuse penumbra or plage region. The coronal temperature of the core was interpreted as due to gyroresonance opacity produced by acceleration of electrons gyrating in a magnetic field. Since the opacity is produced at resonant layers where the frequency matches harmonics of the gyrofrequency, the radio observation could be utilized to measure the coronal magnetic field. Since this simple interferometric observation, the next step for solar astronomers was to use arc second resolution offered by large arrays at $\mathrm{cm}$ wavelengths such as Westerbrock Synthesis Radio Telescope and the Very Large Array, which were primarily built for cosmic radio research. Currently, the Owens Valley Solar Array operating in the range 1-18 GHz and the Nobeyama Radio Heliograph at 17 and $34 \mathrm{GHz}$ are the only solar dedicated radio telescopes. Using these telescopes at multiple wavelengths it is now possible to explore three dimensional structure of sunspot associated radio sources and therefore of coronal magnetic fields. We shall present these measurements at wavelengths ranging from $1.7 \mathrm{~cm}$ to $90 \mathrm{~cm}$ and associated theoretical developments.
\end{abstract}

Keywords. Sun: corona, Sun: magnetic fields, Sun: radio radiation, sunspots

\section{Introduction}

Sunspots appear very bright at cm wavelengths. This property has been known since Covington (1947) observed a sharp decrease in the $10.7 \mathrm{~cm}$ flux during a lunar occultation of a large sunspot group. It was then found in the first spatially resolved observation with a two-element interferometer by Kundu (1959) that solar radio source at $3 \mathrm{~cm}$ has a core-halo structure. The core emission is highly polarized and fully exhibits the coronal temperature and its radiation mechanism had been of a great curiosity while the halo emission was likely to be due to the well-known free-free emission. The most elegant interpretation of the core emission was then provided by Zheleznyakov (1962) in which hot electrons gyrating in strong magnetic fields produce the gyroresonance opacity strong enough to make the corona optically thick at $\mathrm{cm}$ wavelengths. This opacity is high only at the resonant layers where the frequency matches harmonics of the gyrofrequency: $\nu=\Omega_{e}, 2 \Omega_{e}, 3 \Omega_{e}$, and so on. Here $\Omega_{e}=2.8 B \mathrm{MHz}$ is the electron gyrofrequency and $B$ is magnetic field strength in gauss. This simple relation between $\nu$ and $B$ suggests a powerful tool for measuring coronal magnetic fields without complications that arise at other wavelengths and thus opened a new science field of active region coronae. Major progress in this field is reviewed in this article. For more comprehensive reviews, we refer to White \& Kundu (1997), Gary \& Keller (2004) and Lee (2007). 


\section{Studies of Sunspot Emissions}

\subsection{The WSRT and RATAN-600}

The early attempts to measure coronal magnetic field were dominated by the observations with the Westerbork Synthesis Radio Telescope (WSRT) and RATAN-600. The high spatial resolution observations with the WSRT showed that radio sunspots appear in a ring or horseshoe structure in polarization maps (Alissandrakis et al. 1980, Alissandrakis \& Kundu 1982, 1984; Kundu \& Alissandrakis, 1984) which could be due to either dependence of the gyroresonant opacity on the magnetic field orientation or the actual presence of cool plasma $\left(\sim 10^{5} \mathrm{~K}\right)$ overlying the umbra. Alissandrakis \& Kundu (1984) first applied the coronal field extrapolation technique to the interpretation of their center-to-limb observation of a sunspot-associated microwave source to discuss the 3-D magnetic field structure. This characteristic radio morphology found with the WSRT has continued to be discussed with the VLA observations (Vourlidas et al. 1997, Zlotnik et al. 1998). In contrast, observations with the RATAN-600 have emphasized the spectral information available at 5 frequencies with 1-D spatial resolution (Akhmedov et al. 1982; Bogod \& Gelfreikh 1980, Krüger et al. 1986). In these works, extensive calculations of theoretical radio spectra and maps of intensity and polarization were compared with the corresponding observations for agreement. These two types of approaches had remained popular in later studies of sunspot emissions where observations are made at a limited number of frequencies.

\subsection{Imaging spectroscopy with the OVSA}

After the recognition of the importance of multifrequency solar observations, the Owens Valley solar interferometer was converted to frequency-agile operation capable of measuring the solar emission up to 86 frequencies in the range 1-18 GHz (Hurford et al. 1984). As a result spectral observations of a simple sunspot, AR 4741, with the Owens valley Solar Array (OVSA) confirmed that radio spectrum of a sunspot indeed takes the form of the gyroresonant spectrum as predicted by the theory (Lee et al. 1993a). The free-free spectrum was also found when the sunspot went over the limb and the gyroresonant layer was occulted by the limb (Lee et al. 1993b). As expected the radio source reduces in size with increasing frequency, which could then be converted to the spatial distribution of magnetic field strength using the above $\nu-B$ relation. The resulting magnetic field distribution has been compared with theoretical sunspot models to assess the force balance around sunspots (Lee et al. 1993c). Alternatively one can construct a data-cube of 2-D maps along the frequency axis, and then build a set of spectra at each common spatial point. Figure 1 shows the first demonstration of such imaging spectroscopy (Gary \& Hurford 1994). When a spatially resolved spectrum (left panels) indicates the gyroresonant emission, they derived magnetic field strength from the spectral turnover frequency and electron temperature from the optically thick intensity at that location. In the regions where free-free emission dominates the electron density along with an upper limit on the magnetic field was derived. This approach was repeated by Komm et al. (1997) and Bong et al. (2003) for studies of other active regions. The advantage of this approach is that the information of the spatially resolved spectrum at each pixel is so self contained that one does not need to guess the dominant radiation mechanism. These works have established the microwave imaging spectroscopy as a standalone tool for determining the coronal temperature and magnetic field. One aspect overlooked in this procedure was, however, the possible presence of inhomogeneous temperature along the line of sight, which could, though, be appreciated by non-uniformity of the optically thick spectrum. This issue has been addressed in a recent spectral analysis of the joint OVSA-VLA ob- 

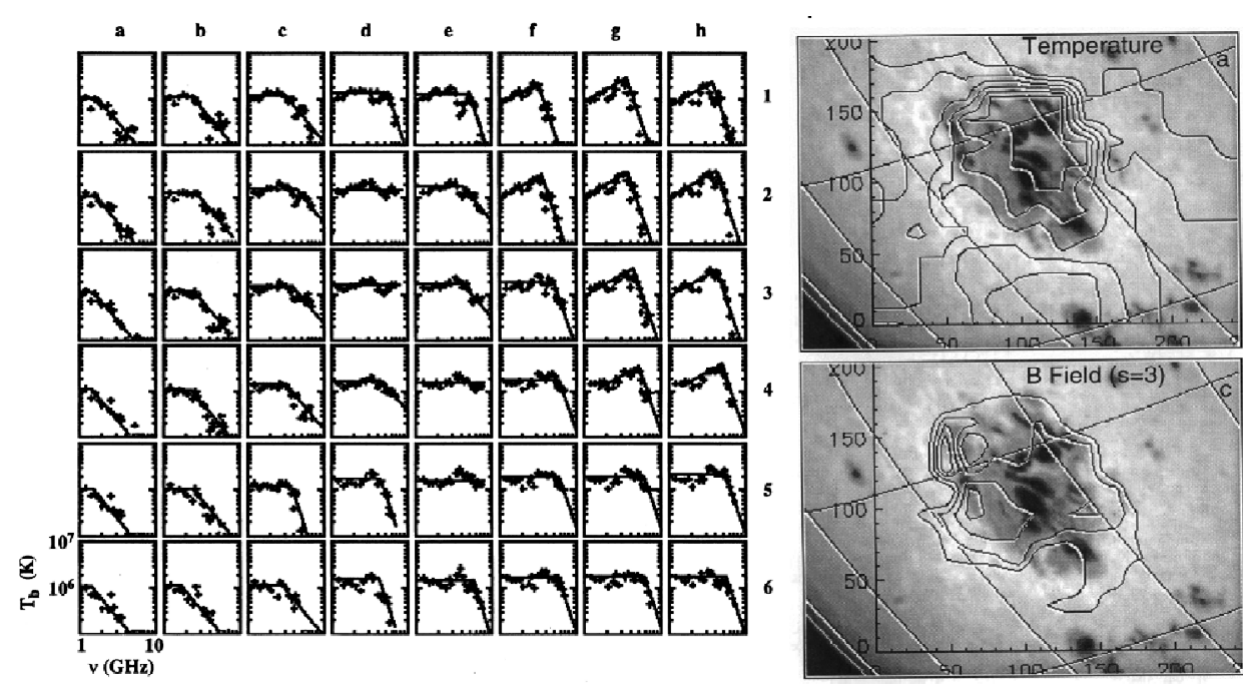

Figure 1. Physical maps from the OVSA imaging spectroscopy. (Left) Brightness temperature spectra obtained at 48 grid points in the data cube represented by the maps. The axis scales for the spectra are all the same, as shown on the spectrum at the lower left. (Right) Physical maps obtained by interpretation of these brightness temperature spectra: electron temperature with contour levels $0.9,1.15,1.4,1.9$ and $2.09 \times 10^{6} \mathrm{~K}$ and total magnetic strength as measured from gyro-resonant spectra assuming the third harmonic everywhere. The contour levels are 200, 400, 600, 800 and 1000 gauss. (from Gary \& Hurford 1994).

servations of AR 10923 where gyroresonant emissions from strong magnetic field region are found to be partly absorbed by over-lying cool materials via the free-free opacity (Tune 2010). As related, O mode polarization found above sunspots (Lee et al. 1993a) and the afore-mentioned ring structure could also be attributed to the inhomogeneous temperature structure over sunspot umbrae.

\subsection{Coronal Magnetic Structures Observing Campaign (CoMStOC)}

The Very Large Array (VLA) is capable of high quality imaging of sunspots but at a few discrete frequencies in which case information on coronal density and temperature obtained at other wavelengths is needed to assist interpretation of the radio data (Schmahl et al. 1982, Strong et al. 1984, Webb et al. 1987). This task has been systematically performed in a series of studies called the CoMStOC in which the plasma electron temperature and emission measures determined from the X-ray and EUV data are used to predict the radio emission expected at the observing frequencies, and these predictions are compared with the radio observations to ascertain the dominant radiation mechanism and derive information on the coronal conditions (Schmelz \& Holman 1991). One example is shown in Figure 2, in which Brosius et al. (2002) used the EUV spectra and images of NOAA 8108 obtained with the Coronal Diagnostic Spectrometer (CDS) and the Extreme-ultraviolet Imaging Telescope (EIT) aboard the SOHO satellite to derive the differential emission measure (DEM) and the electron density for each spatial pixel within both regions. The VLA observations were used to constrain the magnetic scale height and the gross temperature structure of the atmosphere. These, along with the DEM, electron density, and observed radio brightness temperature maps, were then used to derive the height distribution of the coronal magnetic field strength that can reproduce the radio emissions at the observing frequencies for each spatial pixel in the images. Major findings of the CoMStOC include the presence of electric currents in the corona as 

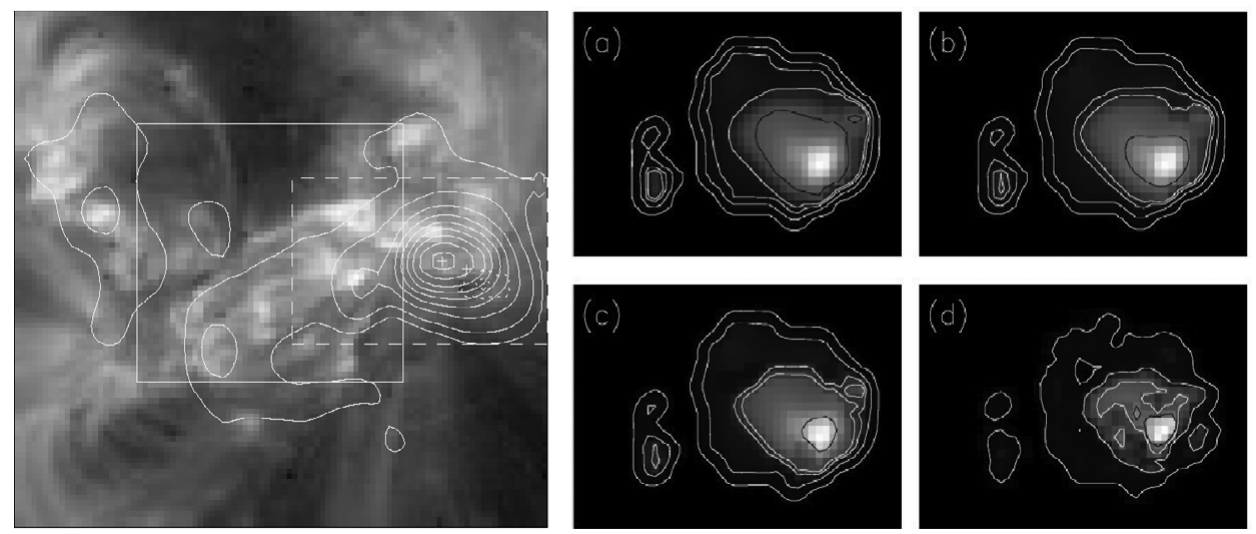

Figure 2. Left: an EIT $195 \AA$ image of AR 8108 on 1997 November 18. The whole field of view is $4^{\prime} \times 4^{\prime}$ and the solid contours correspond to radio brightness temperatures of $0.1,0.25,0.5$, $0.75,1.0,1.25,1.5,1.75$, and $2.0 \times 10^{6} \mathrm{~K}$ in the RCP at $4.866 \mathrm{GHz}$, measured with the VLA. The dashed box of dimension $1.9^{\prime} \times 1.3^{\prime}$ is the area chosen for analysis. Right: the resulting coronal magnetic strengths are shown at heights (a) 5000, (b) 10000, (c) 15000 and (d) 25000 $\mathrm{km}$ above the photosphere. Contour levels are 200, 579, 868, 1005 and 1508 gauss where the last four values correspond, respectively, to $s=3,2$ at $4.866 \mathrm{GHz}$ and $s=3,2$ at $8.450 \mathrm{GHz}$ (from Brosius et al. 2002).

judged from the excess of field strength over the prediction made by the potential field extrapolation, and that of cool material, from the higher predicted brightness temperatures than those observed at $20 \mathrm{~cm}$ (Schmelz et al. 1994).

\subsection{Other Sciences Addressed with the VLA}

The importance of the magnetic topology in coronal temperature structure has been demonstrated by the modeling of high quality VLA maps of a complex active region AR6615 (Lee et al. 1999). The idea is based on the fact that radio maps at multifrequencies represent temperature on different isogauss surfaces and that temperatures measured at two different locations on a field line should be very well correlated. In their result, the correlation between the brightness temperatures measured at 5 and $8 \mathrm{GHz}$ was indeed good for the nonlinear force-free extrapolation and much poorer for the other extrapolations. A more quantitative approach is to adopt a trial heating function and solve a heat transfer equation to determine the temperature along a given field line. Lee et al. (1998) applied several heating functions parameterized in terms of magnetic field, force-free parameter, and current density together with Rosner et al.'s (1978) scaling law to find that the most plausible heating function is proportional to the magnetic field strength at the footpoints, in favor of the hypothesis of coronal heating by nanoflares (Parker 1988).

Gopalswamy (1994, see also Zhang et al. 1998) found radio transient brightenings located near a sunspot umbra, perhaps at the footpoints of an X-ray transient loop observed with Yohkoh, which may indicate small-scale heating. Nindos et al. (2002) used VLA observations to detect spatially resolved oscillations in the total intensity and circular polarization emission of a sunspot-associated gyroresonant source and found that the oscillations are intermittent in time and patchy in space. They interpreted the radio oscillations as due to variations of the location of gyroresonant surfaces with respect to the base of the chromosphere-corona transition region. 


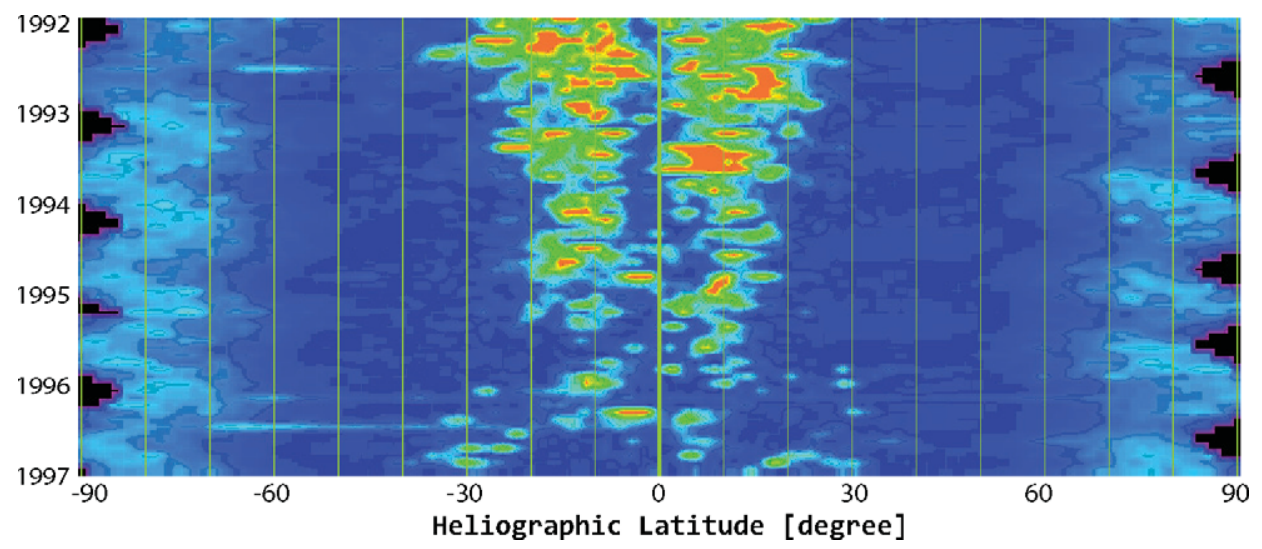

Figure 3. Microwave butterfly diagram constructed with the $17 \mathrm{GHz}$ NoRH data. The bright features are active region belts and polar coronal holes. The dark features are radio counterparts of $\mathrm{H} \alpha$ dark filaments. (Courtesy: K. Shibasaki, 2010).

\subsection{Continuous solar observations with the NoRH}

As a solar dedicated heliograph at $17 \mathrm{GHz}$ and $34 \mathrm{GHz}$, the NoRH has made many important contributions to sunspot emissions, especially, the temporally evolving nature of sunspot emissions as well as individual stationary structures. Shibasaki (1996) reported frequent occurrence of very weak radio bursts (lower than a few s.f.u.) above NOAA 7654 during its entire disk passage, and argued that it is due to short term heating in sunspots. Gelfreikh et al. (1999) measured the circular polarization of several $17 \mathrm{GHz}$ sources as a function of time and found nearly harmonic oscillations with periods mostly between 120-220 s, which are identified with magnetohydrodynamic oscillations. Shibasaki (2001) detected 3-min oscillations in the $17 \mathrm{GHz}$ emission of a sunspot for which he applied the values of density and temperature fluctuations deduced from Solar Ultraviolet Measurements of Emitted Radiation (SUMER) onboard SOHO to find a good agreement with the detected radio oscillation. Investigation of the long term variation of the whole sun emission turns out to be a unique advantage of the NoRH. Shibasaki (1998) produced a radio synoptic map based on the 5-year measurements of $17 \mathrm{GHz}$ daily full disk images, and converted it to a radio butterfly diagram as shown in Figure 3. This radio butterfly diagram shows a similarity with the corresponding diagrams of photospheric magnetic fields and soft X-rays, but reveals that the polar cap brightening gradually expands toward low latitude during the declining phase of the solar cycle 22 .

\section{Concluding Remarks}

Studies of the sunspot emissions at $\mathrm{cm}$ wavelengths have shown that these emissions can serve as an excellent indicator of the coronal magnetic field and temperature. The imaging spectroscopy of sunspots implemented with the OVSA would be one of the best procedures for unambiguous observational determination of these two quantities. The high quality imaging with the VLA and the solar dedicated full-sun imaging with NoRH have produced many important findings of the physical processes in sunspots that include the inhomogeneous structure of plasma, strong kilo gauss magnetic fields, electric currents and magnetohydrodynamic waves present in the sunspot coronae, and various transient activities of sunspot emissions. Sunspot radio emissions are thus particulary suitable for addressing topics related to coronal magnetic field strength and electron temperature. For the future, it is highly desirable to build a next generation solar-dedicated radio telescope 
which should feature as many frequencies as Owens Valley Solar Array offers and as high resolution imaging as the VLA performs. Observing targets may then extend from strong sunspot associated sources to fainter radio sources such as plages, filaments, emerging flux regions and coronal holes to allow an extremely broad range of solar coronal physics to be fully addressed.

The presentation of this paper in the IAU Symposium 273 was possible due to partial support from the NSF grants ATM 0548260, AST 0968672 and NASA LWS grant 09LWSTRT09-0039. J.L. has been supported by NSF grant AST-0908344.

\section{References}

Akhmedov, S. B., Gelfreikh, G. B., Bogod, V. M., \& Korzhavin, A. N. 1982, Solar Phys., 79, 41

Alissandrakis, C. E., Kundu, M. R., \& Lantos, P. 1980, Astron. Astrophys., 82, 30

Alissandrakis, C. E. \& Kundu, M. R. 1982, ApJ Letters, 253, L49

Alissandrakis, C. E. \& Kundu, M. R. 1984, Astron. Astrophys., 139, 271

Bong, S.-C., Lee, J., Gary, D. E., \& Yun, H. S. 2003, J. of the Korean Astron. Soc., 36, S29

Bogod, V. M. \& Gelfreikh, G. B. 1980, Solar Phys., 67, 29

Brosius, J. W., Landi, E., Cook, J. W., Newmark, J. S., Gopalswamy, N., \& Lara, A. 2002, ApJ, 574,453

Covington, A. E. 1947, Nature, 159, 405

Gary, D. E. \& Hurford, G. J. 1994, ApJ, 420, 903

Gary, D. E. \& Keller, C. U. 2004, Solar and Space Weather Radiophysics - Current Status and Future Developments (Dordrecht: Kluwer).

Gelfreikh, G. B., Grechnev, V., Kosugi, T., \& Shibasaki, K. 1999, Solar Phys., 185, 177

Gopalswamy, N., Payne, T. E. W., Schmahl, E. J., Kundu, M. R., Lemen, J. R., Strong, K. T., Canfield, R. C., \& de La Beaujardiere, J. 1994, ApJ, 437, 522

Hurford, G. J., Read, R. B., \& Zirin, H. 1984, Solar Phys., 94, 413

Komm, R. W., Hurford, G. J., \& Gary, D. E. 1997, Astron. Astrophys. Suppl. Ser., 122, 181

Krüger, A., Hildebrandt, J., Bogod, V. M., Korzhavin, A. N., \& Akhmedov, Sh. B. 1986, Solar Phys., 105, 111

Kundu, M. R. 1959, Paris Symposium on Radio Astronomy, Ed. Ronald N. Bracewell, Stanford U. Press, Stanford, p. 222

Kundu, M. R. \& Alissandrakis, C. E. 1984, Solar Phys., 94, 249

Lee, J. 2007, Space Science Reviews, 133, 73

Lee, J., Hurford, G. J., \& Gary, D. E. 1993a, Solar Phys., 144, 45

Lee, J., Gary, D. E., \& Hurford, G. J. 1993b, Solar Phys., 144, 349

Lee, J., Gary, D. E., Hurford, G. J., \& Zirin, H. 1993c, ASPC, 141, 287.

Lee, J., McClymont, A. N., Mikić, Z., White, S. M., \& and Kundu, M. R. 1998, ApJ 501, 853

Lee, J., White, S. M., Kundu, M. R., Mikić, Z., \& McClymont, A. N. 1999, ApJ, 510, 413

Nindos, A., Alissandrakis, C. E., Gelfreikh, G. B., Bogod, V. M., \& Gontikakis, C. 2002, ApJ, 386,658

Parker, E. N. 1988, ApJ, 330, 474

Rosner, R., Tucker, W. H., \& Vaiana, G. S. 1978, ApJ, 220, 643

Schmahl, E. J., Kundu, M. R., Strong, K. T., Bentley, R. D., Smith, J. B., \& Krall, K. R. 1982, Solar Phys., 80, 233

Schmelz, J. T. \& Holman, G. D. 1991, Adv. Space Res., 11, 109

Schmelz, J. T., Holman, G. D., Brosius, J. W., \& Willson, R. F. 1994, ApJ, 434, 786

Shibasaki, K. 1996, Adv. Space Res., 17, 135

Shibasaki, K. 1998, $A S P C, 140,373$

Shibasaki, K. 2001, ApJ, 550, 1113

Strong, K. T., Alissandrakis, C. E., \& Kundu, M. R. 1984, ApJ, 277, 865

Tun, S. 2010, in preparation

Vourlidas, A., Bastian, T. S., \& Aschwanden, M. J. 1997, ApJ, 489, 403 
Webb, D. F., Holman, G. D., Davis, J. M., Kundu, M. R., \& Shevgaonkar, R. K. 1987, ApJ, 315, 716

White, S. M. \& Kundu, M. R. 1997, Solar Phys., 174, 31

Zhang, J., Gopalswamy, N., Kundu, M. R., Schmahl, E. J., \& Lemen, J. R. 1998, Solar Phys., 180, 285

Zheleznyakov, V. V. 1962, Soviet Astr., 6, 3

Zlotnik, E. Ya., White, S. M., \& Kundu, M. R. 1998, ASPC, 155, 135 